\title{
X-ray spectroscopy of current-induced spin-orbit torques and spin accumulation in $\mathrm{Pt} / 3 d$-transition-metal bilayers
}

\author{
C. Stamm,,${ }^{1, *}$ C. Murer,${ }^{1}$ Y. Acremann, ${ }^{2}$ M. Baumgartner, ${ }^{1}$ R. Gort,${ }^{2}$ S. Däster,${ }^{2}$ A. Kleibert, ${ }^{3}$ K. Garello, ${ }^{1}$ J. Feng, \\ M. Gabureac, ${ }^{1,2}$ Z. Chen, ${ }^{4}$ J. Stöhr, ${ }^{4}$ and P. Gambardella ${ }^{1}$ \\ ${ }^{1}$ Department of Materials, ETH Zürich, 8093 Zürich, Switzerland \\ ${ }^{2}$ Laboratory for Solid State Physics, ETH Zürich, 8093 Zürich, Switzerland \\ ${ }^{3}$ Swiss Light Source, Paul Scherrer Institut, 5232 Villigen PSI, Switzerland \\ ${ }^{4}$ Stanford Institute for Materials and Energy Sciences, SLAC National Accelerator Laboratory, 2575 Sand Hill Road, \\ Menlo Park, California 94025, USA
}

(Received 1 April 2019; revised manuscript received 12 July 2019; published 23 July 2019)

\begin{abstract}
An electric current flowing in Pt, a material with strong spin-orbit coupling, leads to spins accumulating at the interfaces by virtue of the spin Hall effect and interfacial charge-spin conversion. We measure the influence of these interfacial magnetic moments onto adjacent $3 d$ transition-metal layers by $\mathrm{x}$-ray absorption spectroscopy and $\mathrm{x}$-ray magnetic circular dichroism in a quantitative and element-selective way, with sensitivity below $10^{-5} \mu_{B}$ per atom. In $\operatorname{Pt}(6 \mathrm{~nm}) / \mathrm{Co}(2.5 \mathrm{~nm})$, the accumulated spins cause a deviation of the Co magnetization direction, which corresponds to an effective spin Hall angle of 0.08 . The spin and orbital magnetic moments of Co are affected in equal proportion by the absorption of the spin current, showing that the transfer of orbital momentum from the recently predicted orbital Hall effect is either below our detection limit, or not directed to the $3 d$ states of Co. For Pt/NM ( $\mathrm{NM}=\mathrm{Ti}, \mathrm{Cr}, \mathrm{Cu}$ ), we find upper limits for the amount of injected spins corresponding to about $3 \times 10^{-6} \mu_{B}$ per atom.
\end{abstract}

DOI: 10.1103/PhysRevB.100.024426

\section{INTRODUCTION}

The coupling of electron spin and charge transport encompasses a broad range of fundamental issues in condensedmatter physics, with straightforward implications for the development of magnetic storage and sensing devices. Spin currents, which mediate the transfer of angular momentum from one material (or part of it) to another, play a central role in this field, as they permit one to manipulate the magnetization, electrical resistance, and heat flow in both metallic and insulating systems [1]. Pure spin currents can be generated via a spin-polarized charge current injection from ferromagnets, spin Hall effect, interfacial charge-spin conversion, spin pumping, or thermal gradients. In most experiments, spin currents are detected electrically [2,3], optically $[4,5]$, in spin pumping measurements [6], or through their influence on the magnetization via spin-orbit torques [7-10]. Recently, a first direct x-ray spectroscopic detection of spin currents was realized in scanning transmission $\mathrm{x}$-ray microscopy by performing AC (current on/off) x-ray magnetic circular dichroism (XMCD) measurements at the $\mathrm{Cu}$ $L_{3}$ edge in a $\mathrm{Co} / \mathrm{Cu}$ nanometer-sized pillar [11]. This measurement revealed a current-induced magnetic signal in the nonmagnetic $\mathrm{Cu}$ layer arising from the spin-polarized current through the heterostructure. The small transient spin signal exhibits a peak at the Fermi level, ascribed to the conduction electrons, and another one that coincides with the static spin signal from $\mathrm{Cu}$ in proximity to $\mathrm{Co}$ [12]. These results motivate

\footnotetext{
*christian.stamm@mat.ethz.ch
}

$\mathrm{x}$-ray spectroscopy investigations of spin currents and their action in systems with high spin-orbit coupling, notably heavy metal/normal metal heterostructures.

Here we report $\mathrm{x}$-ray absorption spectroscopy (XAS) and $\mathrm{XMCD}$ measurements of the current-induced spin accumulation in $\mathrm{Pt} / 3 d$ transition-metal bilayers. In $\mathrm{Pt} / \mathrm{Co}$ samples we detect the accumulated spins through the presence of the spin-orbit torque acting on the magnetic Co layer. The Co spins, initially aligned in the film plane, are slightly tilted out of the plane by an amount proportional to the injection current density. Thereby the ratio of the spin to orbital moment in Co remains unchanged. We further investigated the ability of the spin accumulation to expand from Pt into adjacent layers of the nonmagnetic transition metals $\mathrm{Ti}, \mathrm{Cr}$, and $\mathrm{Cu}$. Here we find that any current-induced XMCD signal at the $L_{3}, L_{2}$ absorption edges of the $3 d$ metal is comparable in size or smaller than our detection limit, which allows us to give an upper limit for the spin current flowing from Pt into the nonmagnetic layer.

\section{EXPERIMENTAL PROCEDURE}

The experiments were performed at the Surface/ Interface:Microscopy (SIM) beamline of the Swiss Light Source at the Paul Scherrer Institut. We measured the absorption of soft $\mathrm{x}$ rays in a thin-film sample by recording the transmitted intensity with a photodiode. This technique allows for highly sensitive XAS and XMCD measurements that are not disturbed by the electric and magnetic fields arising from the injected current used to generate spin currents. After amplification, we split the signal into DC and AC components 


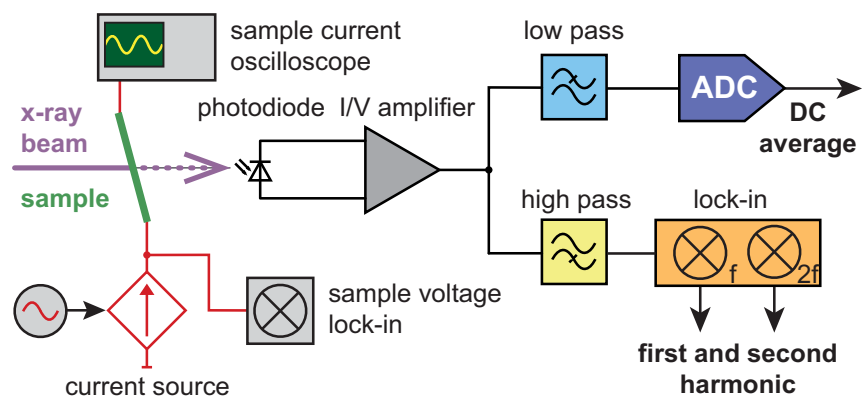

FIG. 1. Schematic drawing of the x-ray transmission setup: it comprises the sample, an in-vacuum photodiode, amplifier, low/high pass filter, ADC, and lock-in amplifier. A sine wave drives the current source, which generates the current that is passed through the sample, monitored by a second lock-in and oscilloscope.

using a combined low pass/high pass filter. The AC signal is processed in a lock-in amplifier which measures the response of the sample in phase with the injected sinusoidal current. A harmonic analysis allows us to separate two effects connected to the first and second harmonic, recorded simultaneously by the lock-in: changes that are directly related to the current are detected in the first harmonic, whereas the second harmonic relates to effects connected to the current squared, e.g.,, the dissipated power in the sample. The current source modulation frequency was set to $50 \mathrm{kHz}$ for all measurements with the exception of $\mathrm{Pt} / \mathrm{Cu}$, for which we used $2030 \mathrm{~Hz}$. The DC response of the photodiode corresponds to the average transmitted intensity and is used to obtain the time-averaged absorption of the sample, resulting in XAS and XMCD spectra. To achieve a reasonably good signal-to-noise ratio in the spectra, multiple photon energy scans were measured and averaged, with the acquisition paused during injection of electrons into the storage ring operating in "top-up" mode. A second lock-in amplifier recorded the applied sinusoidal voltage, and the current through the sample was monitored on an oscilloscope. During the measurements, the vacuum chamber was filled with 100 mbar of He gas in order to dissipate heat from the current-carrying sample. For an illustration of the experimental setup see Fig. 1.

The samples are bilayers consisting of $\mathrm{Pt} / 3 d$ transition metal of either $\mathrm{Ti}, \mathrm{Cr}, \mathrm{Co}$, or $\mathrm{Cu}$. They are sputter-deposited on top of 200-nm-thick $\mathrm{Si}_{3} \mathrm{~N}_{4}$ membranes of $60 \mu \mathrm{m} \times$ $500 \mu \mathrm{m}$ size. A cap layer of $3 \mathrm{~nm} \mathrm{AlO}_{x}$ is used to protect the sample from air. The Pt layers are polycrystalline with (111) texture. Transmission Kikuchi diffraction measurements show that the average grain size is about $15 \mathrm{~nm}$. The rms roughness measured by atomic force microscopy on $\operatorname{Pt}(5 \mathrm{~nm}) / \operatorname{Co}(1 \mathrm{~nm}) / \mathrm{AlO}_{x}$ is about $0.5 \mathrm{~nm}$ and depends on the thickness of the Al layer, whereas the rms roughness of a $15 \mathrm{~nm}$ Pt layer deposited on $\mathrm{Si}_{3} \mathrm{~N}_{4}$ is about $0.3 \mathrm{~nm}$. Despite the low roughness, we cannot exclude minor interdiffusion of $3 d$ elements in $\mathrm{Pt}$ limited to the topmost atomic layers (see, e.g., [13]). The bilayers were directly deposited onto the $\mathrm{Si}_{3} \mathrm{~N}_{4}$ membrane, with few nanometer film thickness as specified in the results section for each sample. On the back of the $\mathrm{Si}_{3} \mathrm{~N}_{4}$ membrane a 150 -nm-thick aluminum layer is deposited which helps to dissipate the heat from the current
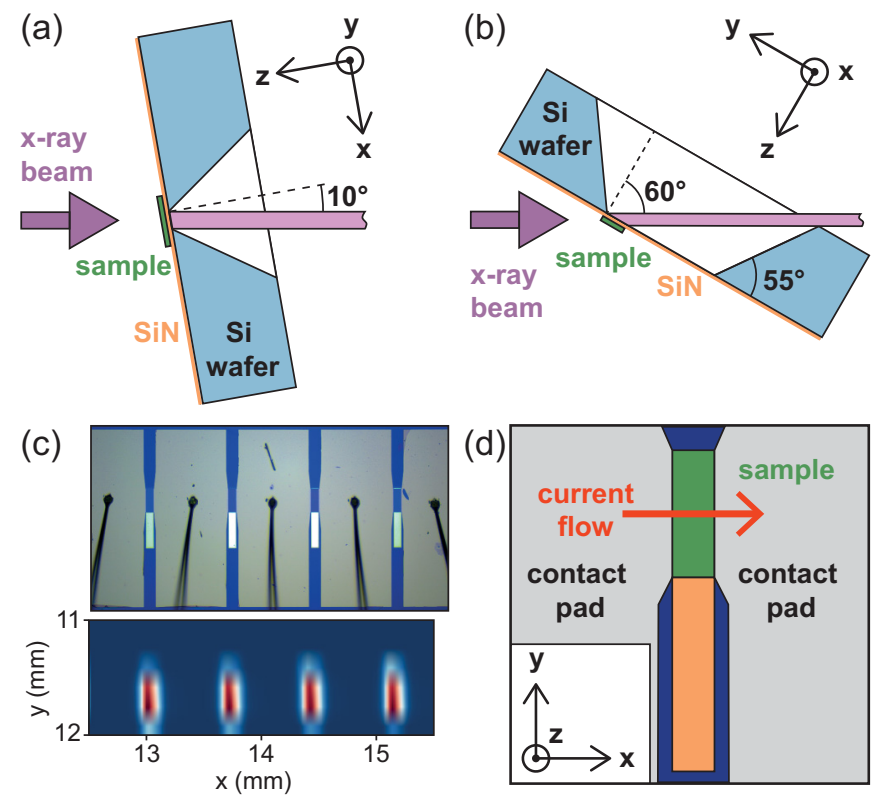

FIG. 2. Sample and measurement geometries for (a) close to perpendicular incidence $\left(10^{\circ}\right)$ mostly sensitive to the perpendicular magnetization component and $(b)$ tilted $\left(\approx 60^{\circ}\right) \mathrm{x}$-ray incidence for detecting in-plane magnetization along $y$. (c) Optical micrograph (above) and $\mathrm{x}$-ray transmitted signal recorded by sample scanning (below) of a sample chip with four current strips. Five wires are attached to the metallic contact pads; in between are the $\mathrm{Si}_{3} \mathrm{~N}_{4}$ membranes with the deposited samples. (d) Close-up sketch of one current strip of $200 \mathrm{~nm}$ width (green), on top of the 500-nm-wide membrane that extends below the sample (orange). The current flow between the contact pads is indicated by the red arrow.

during injection. One Si chip carries four of these membranes, and current strips of 100 or $200 \mu \mathrm{m}$ width are patterned across each one by photolithography. Bonding wires and metallic contact pads are used to electrically connect the current strips, and an external switch box is used to select which structure is subjected to current injection.

Two measurement geometries are employed that have different sensitivities for the spatial magnetization components detected by XMCD. In Fig. 2(a), the tilt angle is $10^{\circ}$ and the measurement is mostly sensitive to the out-of-plane magnetization component $M_{z}$, with a small in-plane $M_{x}$ contribution. This geometry is used when measuring the $\mathrm{Pt} / \mathrm{Co}$ samples with a ferromagnetic Co film. For the second geometry, sketched in Fig. 2(b), the sample is rotated around $x$ until the $\mathrm{x}$-ray beam passing through the strip is almost cut off by the Si frame underneath the membrane. Note that from the membrane fabrication process we have a $55^{\circ}$ cutout angle as a result of the anisotropic etching in $\mathrm{Si}$. In our geometry, the resulting tilt angle is $\approx 60^{\circ}$, which makes the XMCD measurement predominantly sensitive to the in-plane $M_{y}$ component transverse to the current direction, with contributions from $M_{z}$. The actual tilt angle is adjusted for each sample in $1^{\circ}$ steps such that $\mathrm{x}$-rays that did not pass the current strip will not reach the detector. This procedure is needed as the FWHM x-ray beam size was set to $100 \mu \mathrm{m}$ (horizontal) $\times$ $250 \mu \mathrm{m}$ (vertical) [14], which is slightly larger than our current strip sample. The tilted geometry of Fig. 2(b) is used in 
the search for a spin accumulation signal in $\mathrm{Pt} /$ nonmagnetic transition-metal samples. The coordinate system, sketched in Fig. 2, is fixed with the sample that has the current line parallel to $x$ and the surface normal along $z$.

For the successful quantitative evaluation of the XAS data, the correct normalization of the signals is necessary. First, we divide the photodiode signal by the initial intensity as measured on a refocusing mirror, and by the average intensity in the preedge region (photon energy $E<E_{L_{3}}$ ). The x-ray absorption, denoted XAS in our graphs, is then determined by taking the negative logarithm of the normalized transmitted intensity, $-\ln \left(I_{t} / I_{0}\right)$. The DC average intensity and the lock-in AC components are measured simultaneously and at the same scale, and the above-mentioned normalization routine is applied in the same way to all signals, such that they are directly comparable. The static absorption spectrum is corrected for a slope by subtracting a line fitted in the preedge region, which however does not change the scale of the measurement signals.

\section{RESULTS ON Pt/Co}

We begin with describing the measurements on the magnetic bilayer sample $\operatorname{Pt}(6 \mathrm{~nm}) / \operatorname{Co}(2.5 \mathrm{~nm})$. A sinusoidal current runs along $x$ through the sample patterned as a strip of $200 \mu \mathrm{m}$ width and $70 \mu \mathrm{m}$ length, at current densities ranging from 0.79 to $3.78 \times 10^{6} \mathrm{~A} / \mathrm{cm}^{2}$, assuming a homogeneous current distribution throughout the $\mathrm{Pt} / \mathrm{Co}$ structure. As discussed in the Appendix, if one considers the higher resistivity of Pt compared to Co, the actual current density in $\mathrm{Pt}$ is reduced to $\approx 80 \%$ of the values stated here.

The results of the XAS and XMCD measurements at the Co $L_{3}, L_{2}$ absorption edges upon injection at various current densities are displayed in Fig. 3. For Pt/Co we use the measurement geometry drawn in Fig. 2(a) with an almost perpendicular x-ray incidence. A static magnetic field of $42 \mathrm{mT}$ is applied in-plane along the $x$ direction during the measurements, which is sufficient to completely saturate the film and defines the equilibrium magnetization direction. The XMCD measurement is sensitive to the magnetization vector projected onto the $\mathrm{x}$-ray propagation direction, i.e., to both the static in-plane and any out-of-plane component that may arise when current is injected. The signal from the static XMCD component $M_{x}$ results from the projection $\sin \left(10^{\circ}\right)=0.17$ onto the beam direction and is seen in the DC average, and is plotted as a thick black line in Fig. 3(c). Our measurement is, however, mostly sensitive to $M_{z}$ with a projection factor $\cos \left(10^{\circ}\right)=0.98$. The out-of-plane $M_{z}$ component is measured in the lock-in first-harmonic output and is increasing with the injected current [see Fig. 3(b)]. The mechanism behind this behavior is the generation of spin-orbit torques that leads to a change of the equilibrium direction of the magnetization vector $\vec{M}$. In general, spin-orbit torques are classified into two types, according to their direction with respect to the current flow and the sample magnetization: fieldlike and dampinglike torque $[7,8,15]$. Whereas the fieldlike torque leads to a deviation of $\vec{M}$ within the $x y$ plane of the layer, the dampinglike torque can be expressed as a field $B_{\mathrm{DL}} \| z$, effectively pulling the magnetization vector out of the plane.

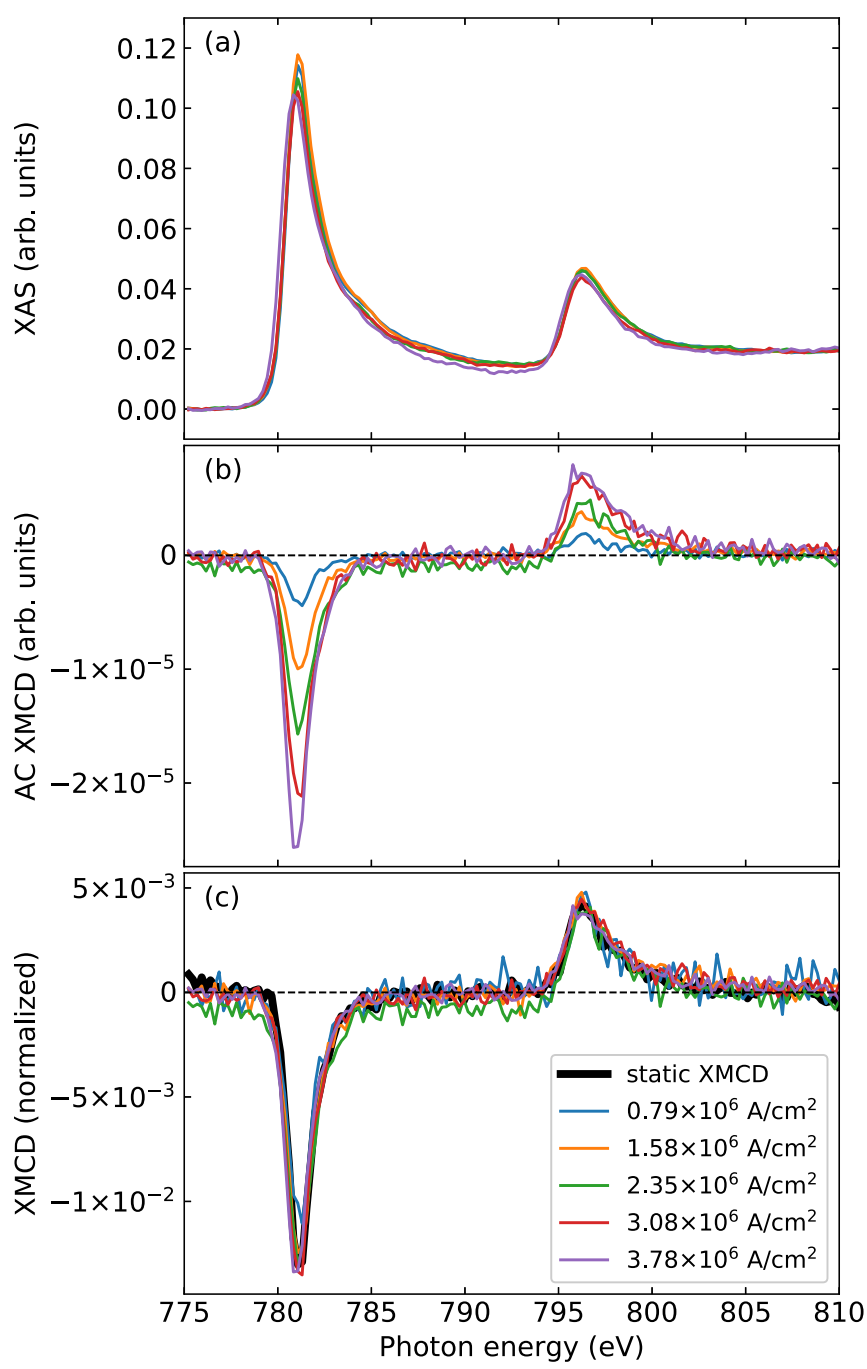

FIG. 3. Co spectra of the Pt/Co sample under the influence of current flow, for given current densities: (a) average XAS, (b) AC$\mathrm{XMCD}$, and (c) AC-XMCD from above rescaled to static XMCD (thick black line).

In Fig. 3(c) we plot the AC-XMCD curves from individual $j$ values, rescaled onto the static XMCD curve. We note that the curves all have the same shape and follow the static one, demonstrating a constant ratio of $L_{3}$ to $L_{2}$ XMCD. This indicates, as will be confirmed in the sum rule analysis below, an unchanged $m_{L} / m_{S}$ ratio of orbital to spin moments in Co. In the recent discussion on the orbital Hall effect (OHE) [16-18] and the orbital Rashba effect [19], an orbital momentum is predicted to accompany the spin Hall effect. The OHE would lead to the accumulation of orbital angular momentum [17], possibly detectable using XMCD. Our data, however, does not show an increased $m_{L} / m_{S}$ ratio, at least in the Co $3 d$ moments at the interface with Pt.

The current dependence of the AC-XMCD is best seen when integrating the respective values at the $L_{3}$ and $L_{2}$ edges. We show the result in Fig. 4(a), together with a linear fit of the induced XMCD. This finding suggests an out-of-plane reorientation of the Co magnetization proportional to the applied current density. For a quantitative analysis of the 


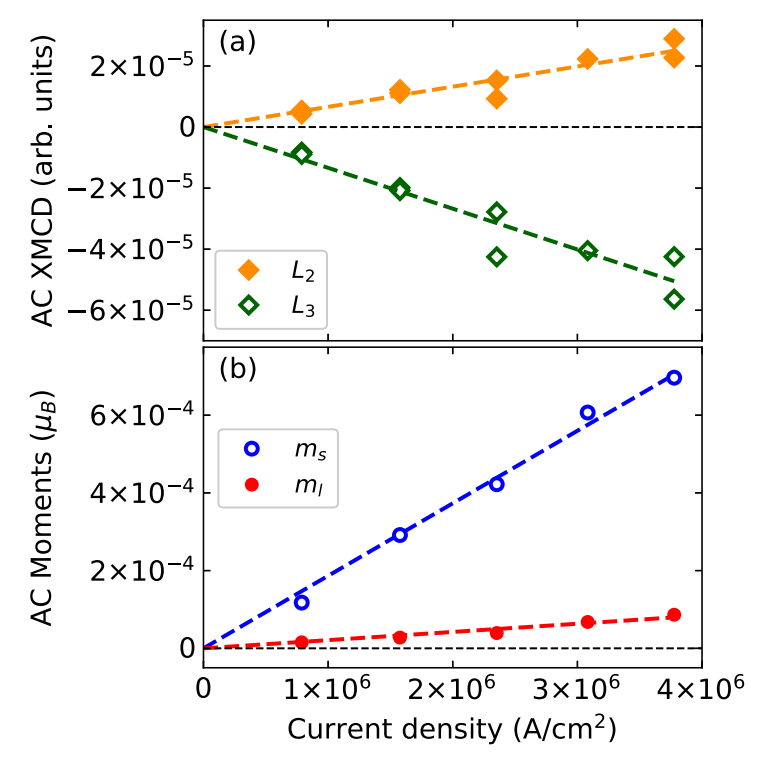

FIG. 4. AC-XMCD versus injected current. (a) Integrated around the Co $L_{2}$ and $L_{3}$ edges, lines denote fits proportional to $j$. (b) Magnetic spin and orbital moment per atom obtained in a sum rules analysis, with line fits.

magnetic moments we use the XMCD sum rules [20-22]. The resulting magnetic moments are plotted as a function of current density in Fig. 4(b), together with their linear fits. For the highest $j=3.78 \times 10^{6} \mathrm{~A} / \mathrm{cm}^{2}$ we found a strong drop of the static magnetic moment to $20 \%-25 \%$ (not shown), meaning that we already heat the sample close to the Curie temperature.

The ratio of the $\mathrm{AC}$ moments to the static moments gives us the out-of-plane deviation angle of the magnetization, $\alpha=$ $0.058^{\circ}$, for $j=10^{7} \mathrm{~A} / \mathrm{cm}^{2}$ assuming homogeneous current density. We now use this value to determine the size of the dampinglike field $B_{\mathrm{DL}}$ that is the origin of $M_{z}$. Together with the sample's out-of-plane anisotropy of $B_{A}=712 \mathrm{mT}$, as determined in a separate harmonic Hall voltage measurement, we get $B_{\mathrm{DL}}=B_{A} \sin (\alpha)=0.72 \mathrm{mT}$, for $j=10^{7} \mathrm{~A} / \mathrm{cm}^{2}$. This value is slightly lower than the $1.17 \mathrm{mT}$ found in harmonic Hall voltage measurements [9] for the same current density and film thicknesses. Further, we determine the spin-orbit torque efficiency to be [23]

$$
\xi_{\mathrm{DL}}=\frac{2 e}{\hbar j} M_{s} t_{\mathrm{Co}} B_{\mathrm{DL}}=0.078
$$

with about $15 \%$ error margins from the determination of the moments, and using $\mu_{0} M_{s}=1.8 \mathrm{~T}$ for Co. The effective spin Hall angle is often taken equal to the spin-orbit torque efficiency, $\theta^{\text {eff }}=\xi_{\mathrm{DL}}$. Alternatively, assuming a drift-diffusion model of the spin Hall effect, the spin-orbit torque efficiency is corrected for the finite Pt thickness [24], giving

$$
\theta^{\text {eff }}=\frac{\xi_{\mathrm{SH}}}{1-1 / \cosh \left(t_{\mathrm{Pt}} / \lambda_{\mathrm{Pt}}\right)},
$$

where $\lambda_{\mathrm{Pt}}$ is the spin diffusion length of Pt, with reported values that range from 1 to $14 \mathrm{~nm}[25,26]$. Finally we note that any fieldlike torque on the Co layer is not detected due to the chosen measurement geometry. It has been shown previously that in $\operatorname{Pt}(6 \mathrm{~nm}) / \operatorname{Co}(2.5 \mathrm{~nm})$ the fieldlike torque is about one order of magnitude smaller than the dampinglike torque [9]. Similarly, we are insensitive to the Oersted field generated by the current through the sample, which is acting along $y$ as the $z$ component averages to zero. An analytic calculation of the Oersted field [27] gives an average value of $0.38 \mathrm{mT}$ within the Co layer.

\section{RESULTS ON Pt/NM bilayers}

Numerous measurements on $\mathrm{Pt} / \mathrm{Co}$ and other magnetic bilayers are present in the current literature, but investigations on $\mathrm{Pt} / \mathrm{NM}$ are rare. Choosing a nonmagnetic layer in contact with $\mathrm{Pt}$ has the advantage of avoiding the direct interaction that the accumulated spins would have with the Co magnetic moment. This is apparent if one compares values of the spin diffusion length: whereas for a single Pt layer at room temperature up to $11 \mathrm{~nm}$ was found [5], this length is reduced to values between 1.1 [28] and $2 \mathrm{~nm} \mathrm{[25]} \mathrm{if} \mathrm{Pt}$ is in contact with Co. Direct XMCD studies at the Pt $L_{3}, L_{2}$ edges [29] would circumvent this difficulty, but the Pt $L$ edges around $11.5 \mathrm{keV}$ photon energy have a lower cross section and reduced XMCD contrast compared to the $3 d$ transition-metal edges, resulting in a sensitivity of $\approx 10^{-3} \mu_{B}$ per Pt atom [30]. Moreover, given the large penetration depth of hard $\mathrm{x}$ rays, one still faces the difficulty of the inhomogeneous spin distribution within the Pt layer: the accumulation on the top interface is balanced by the same accumulation of spins with opposite sign at the bottom interface [31]. To avoid cancellation of the effect in the measurement, the employed technique would need to be sensitive to one layer only. We therefore follow a different approach, in which we add a nonmagnetic (NM) indicator layer of $\mathrm{Ti}, \mathrm{Cr}$, or $\mathrm{Cu}$ on top of Pt. We then perform highly sensitive XMCD measurements on the NM film of the Pt/NM bilayer under current injection.

\section{A. Pt/Ti}

We inject a current of $62 \mathrm{~mA}$ through a $200-\mu \mathrm{m}$-wide strip of the $\operatorname{Pt}(6 \mathrm{~nm}) / \mathrm{Ti}(6 \mathrm{~nm})$ bilayer sample and record XAS and XMCD spectra shown in Fig. 5. Because of the higher resistivity of the Ti layer, the current density in Pt will be increased from $j=2.6 \times 10^{6} \mathrm{~A} / \mathrm{cm}^{2}$ for homogeneous current distribution, to about $j=4.1 \times 10^{6} \mathrm{~A} / \mathrm{cm}^{2}$. The measurement was performed in the geometry drawn in Fig. 2(b). The angle of $60^{\circ}$ ensures the XMCD to be predominantly sensitive to the in-plane magnetic moment, transverse to the current direction, which corresponds to the direction of spins that accumulate due to the spin Hall effect [5].

Even after averaging 56 consecutive energy scans, no dichroic signal could be detected in Ti: the XMCD signal in Fig. 5(a) fluctuates around the zero line, and the X-ray helicity resolved XAS curves, which give the XMCD when subtracted from each other, are almost identical within the noise limit [see Fig. 5(b)]. To estimate the amount of magnetic moment in Ti, we integrated the XMCD signal around the $L_{3}$ and $L_{2}$ edges in a sum rule analysis, and found an upper limit for the induced magnetic moment of $5 \times 10^{-6} \mu_{B}$ per atom. 

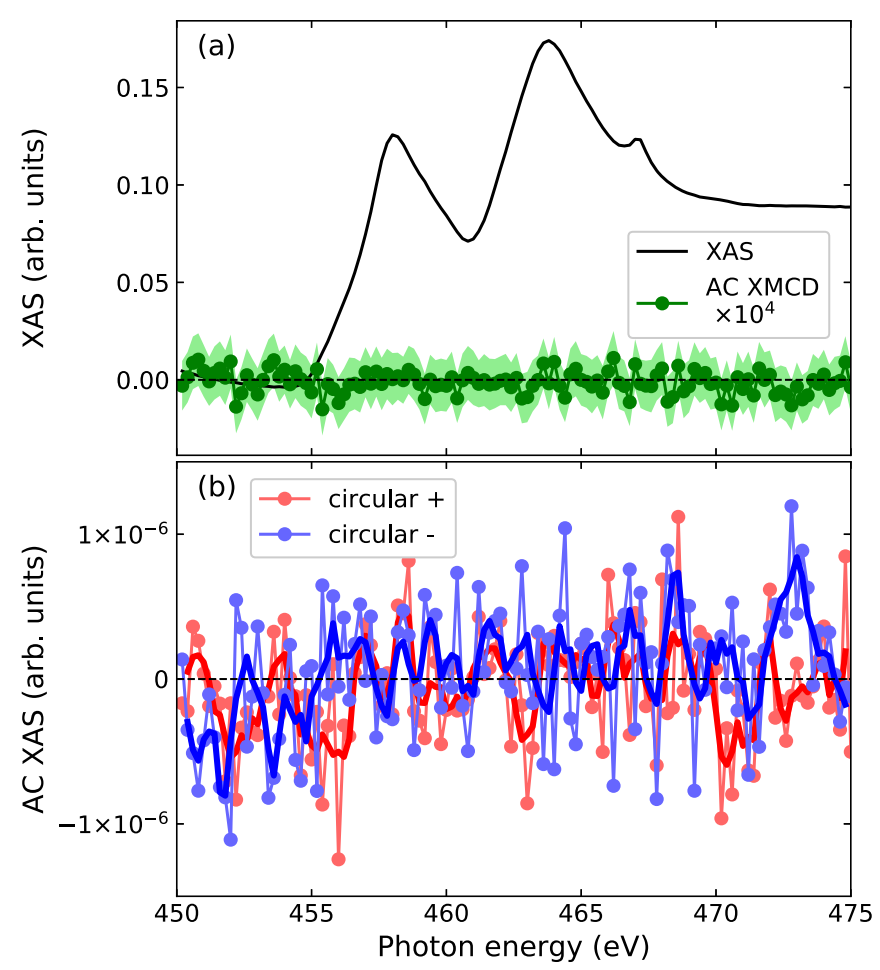

FIG. 5. Pt/Ti bilayer under current injection: (a) XAS (black line) and current-induced XMCD (green dots, standard error marked as light-green band) magnified by the given factor; (b) currentinduced change of the absorption, for circular positive and circular negative $\mathrm{x}$-ray helicity.

\section{B. $\mathbf{P t} / \mathrm{Cr}$}

Next we describe the results of injecting current into a $200-\mu$ m-wide strip of $\operatorname{Pt}(6 \mathrm{~nm}) / \mathrm{Cr}(6 \mathrm{~nm})$. As was recently observed, $\mathrm{Cr}$ has a strong spin-orbit coupling leading to a sizable spin Hall angle of about half that of Pt [32]. In the transmission measurement we, however, integrate the magnetic moment over the whole film thickness, leading to a cancellation of the magnetic signal should there exist a symmetric separation of spins inside the Cr layer. Only an overall spin accumulation that presumably forms at the $\mathrm{Pt} / \mathrm{Cr}$ interface will cause an XMCD signal in Cr. Therefore we are sensitive to the spin-orbit effects in Pt acting on $\mathrm{Cr}$, but not to the intrinsic effects in the $\mathrm{Cr}$ layer.

A current of $72 \mathrm{~mA}$, yielding a current density of $j=$ $3 \times 10^{6} \mathrm{~A} / \mathrm{cm}^{2}$, was injected through $\mathrm{Pt}$, and the sample was measured at an angle of $60^{\circ}$ [see Fig. 2(b)]. A very sensitive measurement was achieved by averaging 144 absorption scans acquired during almost $30 \mathrm{~h}$; the result is shown in Fig. 6. A small hint of a possible spin signal is visible around the $L_{3}$ absorption line, as a dip around $E=579 \mathrm{eV}$ in Fig. 6(a), also visible as differences in the $\mathrm{x}$-ray helicity resolved signals in Fig. 6(b). We notice, however, that noise related fluctuations are of the same amplitude as the presumed spin signal. To further improve the signal-to-noise ratio, we concentrate further measurements on the $\mathrm{Cr} L_{3}$ edge in Fig. 7, the result of averaging 416 spectra. While here we have only half the noise compared to the data shown in Fig. 6, a clear sign of an induced magnetic signal at the $\mathrm{Cr} L_{3}$ edge is still elusive. We
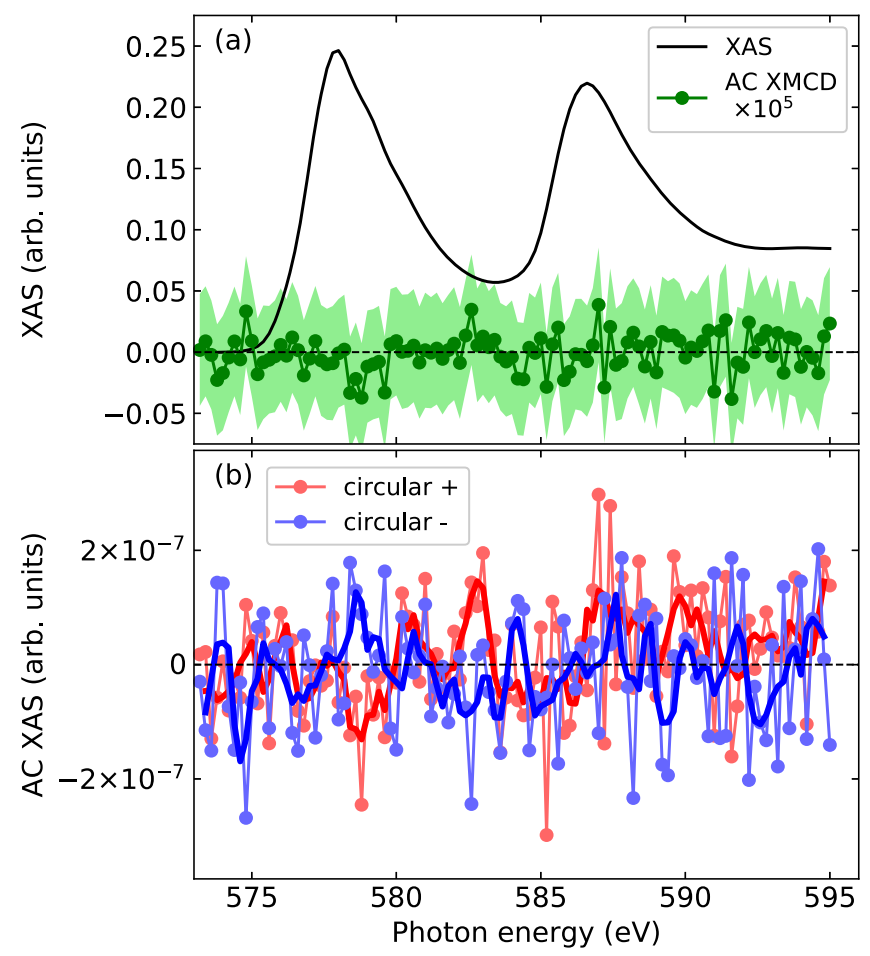

FIG. 6. $\mathrm{Pt} / \mathrm{Cr}$ bilayer under current injection. (a) and (b) as in Fig. 5.

therefore only give an upper limit of a possible spin signal, which amounts to $\approx 2 \times 10^{-6} \mu_{B}$ per $\mathrm{Cr}$ atom, as found in a sum rule integration.

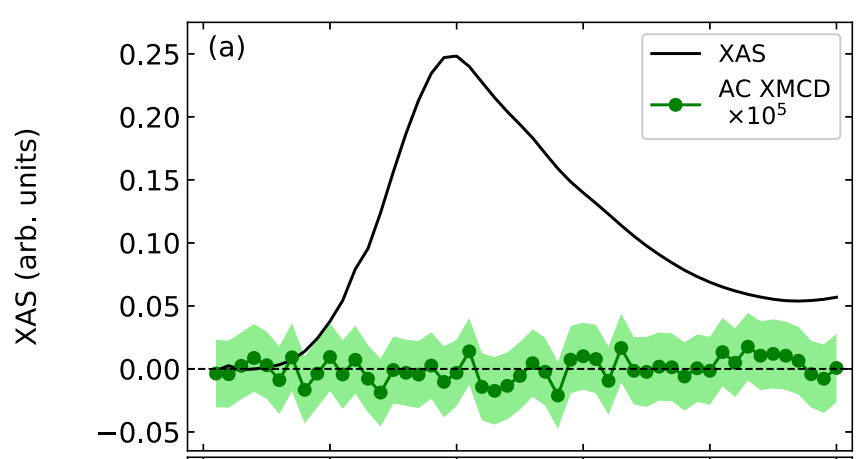

(b)

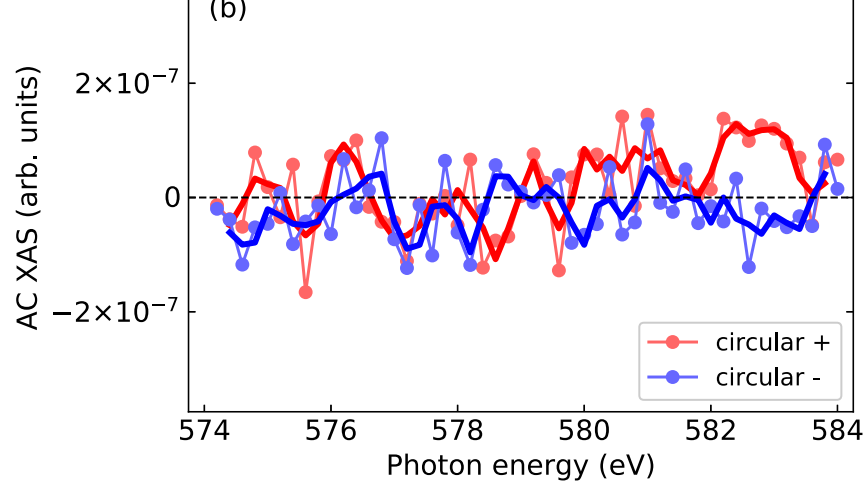

FIG. 7. $\mathrm{Pt} / \mathrm{Cr}$ measurement under current injection, focused around the $\mathrm{Cr} L_{3}$ edge. (a) and (b) as in Fig. 5. 


\section{C. $\mathrm{Pt} / \mathrm{Cu}$}

At last we turn our attention to $\mathrm{Pt} / \mathrm{Cu}$ bilayers. $\mathrm{Cu}$ is a particularly important element for spintronics applications, as it has one of the longest spin diffusion lengths. The excellent conductivity of $\mathrm{Cu}$, however, is a disadvantage for our current-in-plane geometry, as a considerable fraction of the current will actually flow in the $\mathrm{Cu}$ layer and thus does not contribute to spin accumulation in Pt. Measurements have been performed on samples with $\mathrm{Pt}$ thicknesses of 6,10 , and $20 \mathrm{~nm}$, each covered by $10 \mathrm{~nm}$ of $\mathrm{Cu}$, and strips of reduced width of $100 \mu \mathrm{m}$. The results look qualitatively very similar, and we show in Fig. 8 the result of the $\operatorname{Pt}(10 \mathrm{~nm}) / \mathrm{Cu}(10 \mathrm{~nm})$ sample. The $100 \mathrm{~mA}$ current resulted in a current density in the Pt layer of $j=2.6 \times 10^{6} \mathrm{~A} / \mathrm{cm}^{2}$ after accounting for the shunting through $\mathrm{Cu}$, using thin film resistivity values [33].

A very small XMCD signal is visible in Fig. 8(a), also seen as opening of the two curves with opposite X-ray helicity as plotted in Fig. 8(b). A comparison to the XMCD sum rule analysis of $\mathrm{Cu}$ polarized in the proximity of $\mathrm{Co}$ [12] allows us to estimate a magnetic moment of $\approx 1.5 \times 10^{-6} \mu_{B}$ on $\operatorname{Pt}(10$ $\mathrm{nm}) / \mathrm{Cu}(10 \mathrm{~nm})$, with an error margin of the same size. Similar results come from measurements on a $\mathrm{Pt}(20 \mathrm{~nm}) / \mathrm{Cu}(10$ $\mathrm{nm}$ ) sample shown in Figs. 8(c) and 8(d). Without judging the significance of the signal, we conclude that there may be signs of spins injected from the accumulation in $\mathrm{Pt}$, but their moments average in the $\mathrm{Cu}$ layer to less than $\approx 3 \times 10^{-6} \mu_{B}$ per atom.

\section{DISCUSSION AND SUMMARY}

Summarizing our measurements on $\mathrm{Pt} / \mathrm{Co}$ under current injection, we found that the magnetization of Co reorients itself from purely in-plane into a new equilibrium position with a small perpendicular component. This process is described by a dampinglike field, a consequence of the current-induced accumulation of spins at the $\mathrm{Pt} / \mathrm{Co}$ interface. The quantitative analysis of our XMCD measurements reveals a spin Hall angle of $\theta^{\text {eff }}=0.08$. In addition, our XMCD analysis shows a constant $m_{L} / m_{S}$ ratio in Co that is independent of the injected current density.

Next we exchanged Co with a nonmagnetic $3 d$ transition metal and measure with high accuracy the amount of spins that emerge in $\mathrm{Ti}, \mathrm{Cr}$, or $\mathrm{Cu}$. Any signal of accumulated spins is, however, so small that we can only give upper limits of spin accumulation, with the exception of $\mathrm{Cu}$ which may exhibit a moment of $\approx 1.5 \times 10^{-6} \mu_{B}$ although with error bars of the same size. A similar conclusion was reported in an attempt to measure the injected spins in a lateral spin valve, for which the authors could not reveal magnetic contrast on the nonmagnetic $\mathrm{Cu}$ electrodes but gave an upper limit of $10^{-4} \mu_{B}$ XMCD contrast [34]. Only in the measurements reported on a $\mathrm{Co} / \mathrm{Cu}$ nanopillar, a small but measurable spin moment of $3 \times 10^{-5} \mu_{B}$ was detected [11]. In the nanopillar the current is forced to flow perpendicular, from $\mathrm{Co}$ into $\mathrm{Cu}$, and thus the spins are transported into $\mathrm{Cu}$ with very high efficiency.

We now compare the result on $\mathrm{Pt} / \mathrm{Cu}$ presented here with our previous magneto-optical measurement of the spin Hall effect induced spin accumulation in a single Pt layer [5]. At our current density, one expects an accumulation of $1.3 \times$
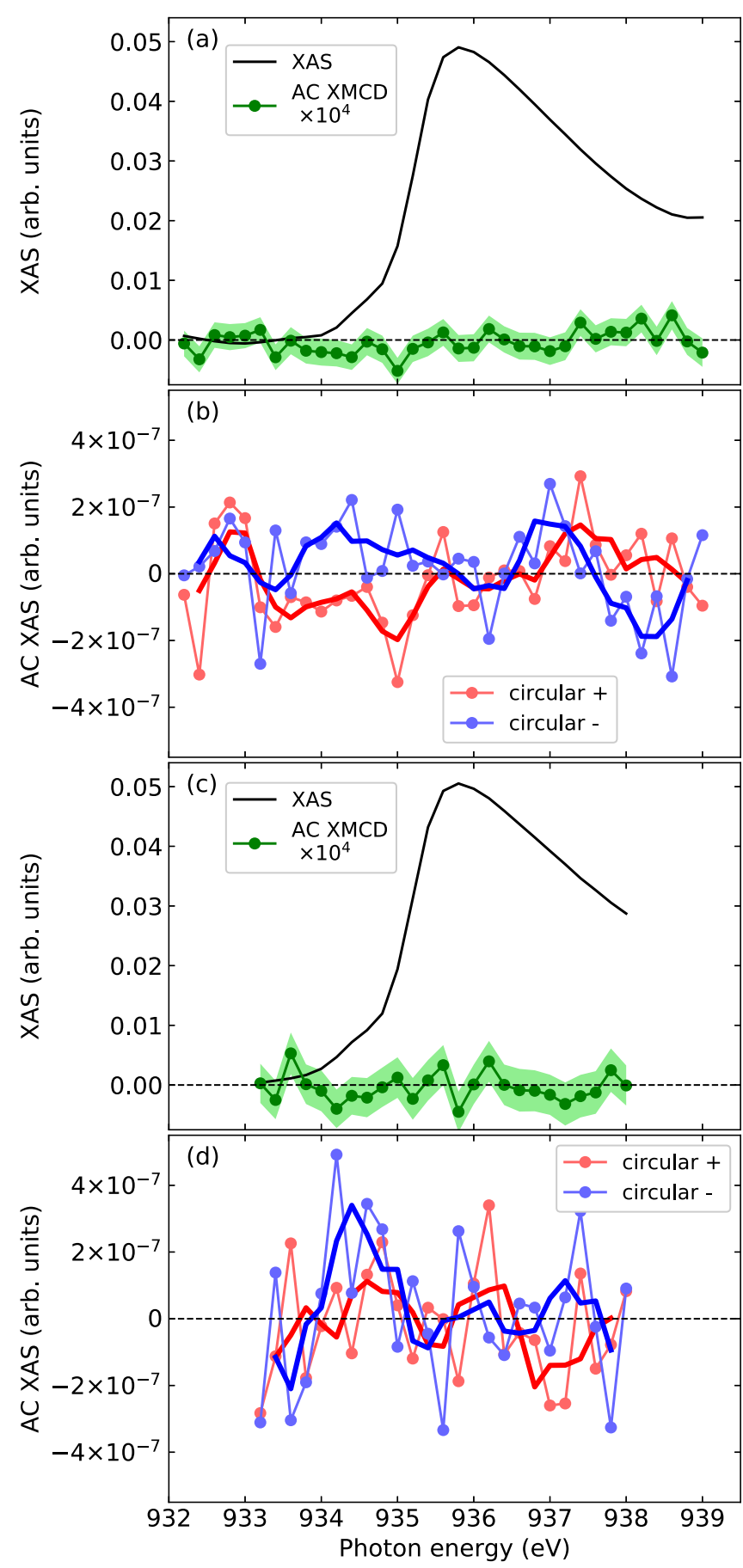

FIG. 8. XAS and XMCD of $\mathrm{Pt} / \mathrm{Cu}$, measured at the $\mathrm{Cu} L_{3}$ edge. (a) $\mathrm{Pt}(10 \mathrm{~nm}) / \mathrm{Cu}(10 \mathrm{~nm})$ subjected to a current of $100 \mathrm{~mA}$ : XAS (black line) and current-induced XMCD (green dots) magnified by $10^{4}$, with XMCD standard error as a light-green band. (b) Currentinduced change of the absorption, for circular positive and circular negative x-ray helicity. (c), (d) Same graphs as (a) and (b), for $\mathrm{Pt}(20 \mathrm{~nm}) / \mathrm{Cu}(10 \mathrm{~nm})$ subjected to a current of $80 \mathrm{~mA}$.

$10^{-5} \mu_{B}$ at the surface of Pt, for thickness $t_{\mathrm{Pt}} \gg \lambda_{\mathrm{Pt}}=11 \mathrm{~nm}$. For the $10 \mathrm{~nm} \mathrm{Pt}$ thickness used here, this value would be reduced to $\approx 4 \times 10^{-6} \mu_{B}$ using the correction for finite $\mathrm{Pt}$ thickness $[24,25]$ but with the spin diffusion length of a single Pt layer [5] of $\lambda_{\mathrm{Pt}}=11 \mathrm{~nm}$. Experimentally we determined 
the moment in $\mathrm{Cu}$ to be lower than $\approx 3 \times 10^{-6} \mu_{B}$ for the 10 $\mathrm{nm} \mathrm{Pt} / \mathrm{Cu}$ sample, which confirms that we are in the range of detecting the magnetic moment. An unknown factor, however, is the role of the $\mathrm{Pt} / \mathrm{Cu}$ interface, which may introduce spin loss due to electron scattering, leading to a further reduction of detectable spins in the $\mathrm{Cu}$ layer. As we see from the correction for finite Pt thickness, a thicker Pt layer would help increasing the spin accumulation. We additionally measured a $\mathrm{Pt}(20 \mathrm{~nm}) / \mathrm{Cu}(10 \mathrm{~nm})$ sample with twice the Pt thickness, and would expect roughly a twofold increase of the AC-XMCD signal. However, the $20 \mathrm{~nm} \mathrm{Pt} / \mathrm{Cu}$ has a lower current density in Pt of $1.8 \times 10^{6} \mathrm{~A} / \mathrm{cm}^{2}$. Overall we would expect the AC$\mathrm{XMCD}$ to be similar in size for the two $\mathrm{Pt} / \mathrm{Cu}$ samples, which is indeed found comparing panels (a) and (c) in Fig. 8.

Finally we estimate the influence of the Oersted field, which lies along the same axis as the presumed accumulated moment in the $\mathrm{Pt} / \mathrm{NM}$ current strips. The calculation for $\mathrm{Pt}(10 \mathrm{~nm}) / \mathrm{Cu}(10 \mathrm{~nm})$ gives a value of $B_{\mathrm{Oe}}=0.09 \mathrm{mT}$, which leads to an induced magnetic moment $M=\chi B / \mu_{0}$, with $\chi=-9.63 \times 10^{-6}$ being the magnetic susceptibility of $\mathrm{Cu}$ [35]. In $\mathrm{Pt} / \mathrm{Cu}$, the Oersted field will thus induce a magnetic moment corresponding to $\approx 10^{-9} \mu_{B}$ per atom, well below our experimental sensitivity and far lower than the presumed spin accumulation signal. Analogous numbers are found for $\mathrm{Pt} / \mathrm{Ti}$ and $\mathrm{Pt} / \mathrm{Cr}$, and we thus conclude that contributions from the Oersted field can be neglected in Pt/NM bilayers.

\section{ACKNOWLEDGMENTS}

We thank C. O. Avci for fruitful discussions and T. Bähler, L. Debenjak, and P. Schifferle for technical assistance. We acknowledge the Paul Scherrer Institut, Villigen, Switzerland for provision of synchrotron radiation beamtime at the SIM beamline of the Swiss Light Source, and the Swiss National
Science Foundation for financial support through Grants No. 200021-153404 and No. 200020-172775.

\section{APPENDIX: EFFECTIVE CURRENT DENSITY IN Pt LAYER}

When injecting current into a Pt/NM bilayer sample, both layers will conduct a fraction of the current according to their electrical resistivity and thickness. Here we estimate the individual current density inside each layer of the $\mathrm{Pt} / \mathrm{Co}$ sample by assuming two resistors in parallel, $R_{\mathrm{Pt}}$ and $R_{\mathrm{Co}}$. The voltage across each resistor is identical, $U_{\mathrm{Pt}}=U_{\mathrm{Co}}=R_{\mathrm{Pt}} I_{\mathrm{Pt}}=$ $R_{\mathrm{Co}} I_{\mathrm{Co}}$, whereas the total current is given by the sum of the currents through each layer, $I=I_{\mathrm{Pt}}+I_{\mathrm{Co}}$. We get the current ratio as the inverse of the resistance ratio

$$
r=\frac{I_{\mathrm{Pt}}}{I_{\mathrm{Co}}}=\frac{R_{\mathrm{Co}}}{R_{\mathrm{Pt}}}=\frac{\rho_{\mathrm{Co}}}{\rho_{\mathrm{Pt}}} \frac{t_{\mathrm{Pt}}}{t_{\mathrm{Co}}},
$$

using the definition of the resistance $R_{i}=\rho_{i} l /\left(t_{i} w\right)$, with identical strip length $l$ and width $w$ for both layers, and independent resistivity $\rho_{i}$ and thickness $t_{i}$ values. For $\operatorname{Pt}(6 \mathrm{~nm}) / \mathrm{Co}(2.5 \mathrm{~nm})$ we use values found in a separate fourpoint measurement on Hall bar structures, $\rho_{\mathrm{Pt}}=255 \mathrm{n} \Omega \mathrm{m}$ and $\rho_{\mathrm{Co}}=600 \mathrm{n} \Omega \mathrm{m}$, resulting in $r=5.65$.

The fraction of current in each layer is given by

$$
\frac{I_{\mathrm{Pt}}}{I}=\frac{r}{1+r}=0.85 \quad \text { and } \quad \frac{I_{\mathrm{Co}}}{I}=\frac{1}{1+r}=0.15 .
$$

The relative current density in Pt then becomes

$$
\frac{j_{\mathrm{Pt}}}{j}=\frac{I_{\mathrm{Pt}}}{I} \frac{t_{\mathrm{Pt}}+t_{\mathrm{Co}}}{t_{\mathrm{Pt}}}=\frac{r}{1+r} \frac{8.5 \mathrm{~nm}}{6 \mathrm{~nm}}=1.20,
$$

or a $20 \%$ increase compared to the current density that was calculated assuming a homogeneous distribution.
[1] Spin Current, edited by S. Maekawa, S. Valenzuela, E. Saitoh, and T. Kimura (Oxford University Press, Oxford, 2012).

[2] S. O. Valenzuela and M. Tinkham, Direct electronic measurement of the spin Hall effect, Nature (London) 442, 176 (2006).

[3] Y. Niimi and Y. Otani, Reciprocal spin Hall effects in conductors with strong spin-orbit coupling: A review, Rep. Prog. Phys. 78, 124501 (2015).

[4] Y. K. Kato, R. C. Myers, A. C. Gossard, and D. D. Awschalom, Observation of the spin Hall effect in semiconductors, Science 306, 1910 (2004).

[5] C. Stamm, C. Murer, M. Berritta, J. Feng, M. Gabureac, P. M. Oppeneer, and P. Gambardella, Magneto-Optical Detection of the Spin Hall Effect in Pt and W Thin Films, Phys. Rev. Lett. 119, 087203 (2017).

[6] E. Saitoh, M. Ueda, H. Miyajima, and G. Tatara, Conversion of spin current into charge current at room temperature: Inverse spin-Hall effect, Appl. Phys. Lett. 88, 182509 (2006).

[7] K. Garello, I. M. Miron, C. O. Avci, F. Freimuth, Y. Mokrousov, S. Blugel, S. Auffret, O. Boulle, G. Gaudin, and P. Gambardella, Symmetry and magnitude of spin-orbit torques in ferromagnetic heterostructures, Nat. Nanotechnol. 8, 587 (2013).
[8] J. Kim, J. Sinha, M. Hayashi, M. Yamanouchi, S. Fukami, T. Suzuki, S. Mitani, and H. Ohno, Layer thickness dependence of the current-induced effective field vector in $\mathrm{Ta}|\mathrm{CoFeB}| \mathrm{MgO}$, Nat. Mater. 12, 240 (2013).

[9] C. O. Avci, K. Garello, M. Gabureac, A. Ghosh, A. Fuhrer, S. F. Alvarado, and P. Gambardella, Interplay of spin-orbit torque and thermoelectric effects in ferromagnet/normal-metal bilayers, Phys. Rev. B 90, 224427 (2014).

[10] M. Baumgartner, K. Garello, J. Mendil, C. O. Avci, E. Grimaldi, C. Murer, J. Feng, M. Gabureac, C. Stamm, Y. Acremann, S. Finizio, S. Wintz, J. Raabe, and P. Gambardella, Spatially and time-resolved magnetization dynamics driven by spin-orbit torques, Nat. Nanotechnol. 12, 980 (2017).

[11] R. Kukreja, S. Bonetti, Z. Chen, D. Backes, Y. Acremann, J. A. Katine, A. D. Kent, H. A. Dürr, H. Ohldag, and J. Stöhr, X-Ray Detection of Transient Magnetic Moments Induced by a Spin Current in Cu, Phys. Rev. Lett. 115, 096601 (2015).

[12] M. G. Samant, J. Stöhr, S. S. P. Parkin, G. A. Held, B. D. Hermsmeier, F. Herman, M. van Schilfgaarde, L.-C. Duda, D. C. Mancini, N. Wassdahl, and R. Nakajima, Induced Spin Polarization in $\mathrm{Cu}$ Spacer Layers in $\mathrm{Co} / \mathrm{Cu}$ Multilayers, Phys. Rev. Lett. 72, 1112 (1994). 
[13] P. Gambardella, M. Blanc, L. Bürgi, K. Kuhnke, and K. Kern, Co growth on $\mathrm{Pt}(997)$ : From monatomic chains to monolayer completion, Surf. Sci. 449, 93 (2000).

[14] G. Olivieri, A. Goel, A. Kleibert, and M. A. Brown, Effect of x-ray spot size on liquid jet photoelectron spectroscopy, J. Synchrotron Radiat. 22, 1528 (2015).

[15] P. M. Haney, H.-W. Lee, K.-J. Lee, A. Manchon, and M. D. Stiles, Current induced torques and interfacial spin-orbit coupling: Semiclassical modeling, Phys. Rev. B 87, 174411 (2013).

[16] H. Kontani, T. Tanaka, D. S. Hirashima, K. Yamada, and J. Inoue, Giant Orbital Hall Effect in Transition Metals: Origin of Large Spin and Anomalous Hall Effects, Phys. Rev. Lett. 102, 016601 (2009).

[17] D. Go, D. Jo, C. Kim, and H.-W. Lee, Intrinsic Spin and Orbital Hall Effects from Orbital Texture, Phys. Rev. Lett. 121, 086602 (2018).

[18] D. Jo, D. Go, and H.-W. Lee, Gigantic intrinsic orbital Hall effects in weakly spin-orbit coupled metals, Phys. Rev. B 98, 214405 (2018).

[19] X. Chen, Y. Liu, G. Yang, H. Shi, C. Hu, M. Li, and H. Zeng, Giant antidamping orbital torque originating from the orbital Rashba-Edelstein effect in ferromagnetic heterostructures, Nat. Commun. 9, 2569 (2018).

[20] B. T. Thole, P. Carra, F. Sette, and G. van der Laan, X-Ray Circular Dichroism as a Probe of Orbital Magnetization, Phys. Rev. Lett. 68, 1943 (1992).

[21] P. Carra, B. T. Thole, M. Altarelli, and X. Wang, X-Ray Circular Dichroism and Local Magnetic Fields, Phys. Rev. Lett. 70, 694 (1993).

[22] C. T. Chen, Y. U. Idzerda, H.-J. Lin, N. V. Smith, G. Meigs, E. Chaban, G. H. Ho, E. Pellegrin, and F. Sette, Experimental Confirmation of the X-Ray Magnetic Circular Dichroism Sum Rules for Iron and Cobalt, Phys. Rev. Lett. 75, 152 (1995).

[23] M.-H. Nguyen, D. C. Ralph, and R. A. Buhrman, Spin Torque Study of the Spin Hall Conductivity and Spin Diffusion Length in Platinum Thin Films with Varying Resistivity, Phys. Rev. Lett. 116, 126601 (2016).

[24] L. Liu, T. Moriyama, D. C. Ralph, and R. A. Buhrman, SpinTorque Ferromagnetic Resonance Induced by the Spin Hall Effect, Phys. Rev. Lett. 106, 036601 (2011).
[25] J. Sinova, S. O. Valenzuela, J. Wunderlich, C. H. Back, and T. Jungwirth, Spin Hall effects, Rev. Mod. Phys. 87, 1213 (2015).

[26] E. Sagasta, Y. Omori, M. Isasa, M. Gradhand, L. E. Hueso, Y. Niimi, Y. C. Otani, and F. Casanova, Tuning the spin Hall effect of Pt from the moderately dirty to the superclean regime, Phys. Rev. B 94, 060412(R) (2016).

[27] M. Hayashi, J. Kim, M. Yamanouchi, and H. Ohno, Quantitative characterization of the spin-orbit torque using harmonic Hall voltage measurements, Phys. Rev. B 89, 144425 (2014).

[28] C. O. Avci, K. Garello, A. Ghosh, M. Gabureac, S. F. Alvarado, and P. Gambardella, Unidirectional spin Hall magnetoresistance in ferromagnet/normal metal bilayers, Nat. Phys. 11, 570 (2015).

[29] W. Grange, M. Maret, J.-P. Kappler, J. Vogel, A. Fontaine, F. Petroff, G. Krill, A. Rogalev, J. Goulon, M. Finazzi, and N. B. Brookes, Magnetocrystalline anisotropy in (111)CoPt3 thin films probed by x-ray magnetic circular dichroism, Phys. Rev. B 58, 6298 (1998).

[30] S. Geprägs, S. Meyer, S. Altmannshofer, M. Opel, F. Wilhelm, A. Rogalev, R. Gross, and S. T. B. Goennenwein, Investigation of induced $\mathrm{Pt}$ magnetic polarization in $\mathrm{Pt} / \mathrm{Y}_{3} \mathrm{Fe}_{5} \mathrm{O}_{12}$ bilayers, Appl. Phys. Lett. 101, 262407 (2012).

[31] S. Zhang, Spin Hall Effect in the Presence of Spin Diffusion, Phys. Rev. Lett. 85, 393 (2000).

[32] C. Du, H. Wang, F. Yang, and P. C. Hammel, Systematic variation of spin-orbit coupling with $d$-orbital filling: Large inverse spin Hall effect in $3 d$ transition metals, Phys. Rev. B 90, 140407(R) (2014).

[33] S. Dutta, K. Sankaran, K. Moors, G. Pourtois, S. V. Elshocht, J. Bömmels, W. Vandervorst, Z. Tőkei, and C. Adelmann, Thickness dependence of the resistivity of platinum-group metal thin films, J. Appl. Phys. 122, 025107 (2017).

[34] O. Mosendz, G. Mihajlović, J. E. Pearson, P. Fischer, M.-Y. Im, S. D. Bader, and A. Hoffmann, Imaging of lateral spin valves with soft x-ray microscopy, Phys. Rev. B 80, 104439 (2009).

[35] J. F. Schenck, The role of magnetic susceptibility in magnetic resonance imaging: MRI magnetic compatibility of the first and second kinds, Med. Phys. 23, 815 (1996). 